\title{
CORRESPONDENCE
}

Dear Sir,

\section{Language and Law in Tanzania}

Mr. Harries's thoughtful and helpful article, ${ }^{1}$ which practically becomes a paragraph-by-paragraph commentary on my earlier article, is of course quite right from the point of view of an academic linguist, though it does, through some attitude of hostility, miss some of the points I made.

No one, of course, could ever be so naive as to suggest that any language is pure, and not a constantly changing amalgam. But it is the rate of absorption that is culturally-and politically-noteworthy in the case of present-day Swahili. Whereas Mr. Harries seems prepared to accept the mental attitudes-of pride, prestige and laziness, sometimes called neo-colonialism-of many of the newly elevated Tanzanians, and the resulting rapid "bastardization" of the language to which I refer (many indeed would prefer to see English itself remain as the official and national language), nevertheless he is critical of the attempt of the Committee (and of many others like it) to speed up the process of modernization and enlargement of Swahili.

He also implies that the government is naive enough to believe that it can by legislation force the development of language along exact lines. Were this true, it would be right for the academic to scold, and to smile. But of course it is not so. Of course this law dictionary, and all others being prepared, are merely efforts of goodwill by dedicated people who hope thereby to assist and encourage development. All change must be tentative. And though the English linguists who have "studied" and thereby put their own stamp on Swahili may look with some disdain on the products, they are surprisingly acceptable to those educated and sophisticated Swahilis who really know their own language.

Mr. Harries quotes the simple and well-known rules of language development, as if they were rules which cannot or should not be departed from. In doing this he speaks as a distant academic with little knowledge of or feel for the current social and political realities of Tanzania.

It is so easy to be supercilious and critical, and therefore destructive, of attempts by newly independent states to develop their own indigenous institutions and cultures, and so difficult to be sympathetic, and more so, encouraging and constructive, especially when one belongs to the language and culture which those states are in the process of rejecting.

University College, Dar-es-Salaam.

(Professor) A. B. Weston

${ }^{2}[1966]$ J.A.L. 164 . 